\title{
Giant Pleomorphic Adenoma of the Nasal Septum
}

\author{
Tekin Baglam • Cengiz Durucu • Cengiz Cevik • \\ Kemal Bakir • Arzu Oz • Muzaffer Kanlikama
}

Received: 15 July 2009/Accepted: 31 January 2010/Published online: 10 April 2011

(C) Association of Otolaryngologists of India 2011

\begin{abstract}
Pleomorphic adenoma is the most common benign tumor of the salivary glands, mainly arising from major salivary glands such as parotis and submandibular gland. In rare cases, however, pleomorphic adenoma presents in various unusual sites such as the nasal cavity, paranasal sinuses, hypopharynx, pharynx, larynx, trachea and lacrimal glands. We present a rare case of 80 -year-old woman with pleomorphic adenoma of the nasal cavity.
\end{abstract}

Keywords Plemorphic adenoma $\cdot$ Nasal $\cdot$ Septum

\section{Introduction}

Pleomorphic adenoma is the most common benign salivary gland tumor. Parotid gland constitutes $60 \%$ of all pleomorphic adenomas while minor salivary glands constitutes only $8 \%$ of them [1]. Pleomorphic adenomas of the minor salivary glands can develop in any site where the minor salivary glands exist such as nasal cavity, paranasal sinuses, hypopharynx, pharynx, larynx, trachea and lacrimal glands [2].

Intranasal pleomorphic adenomas are quite rare, slow growing tumors usually originating from mucosa of nasal septum, although minor salivary glands located on lateral nasal wall [3]. We report a delayed case of pleomorphic adenoma of the nasal septum presented with giant mass causing nasal deformity and total obstruction of nasal

T. Baglam ( $₫)$ C C. Durucu · C. Cevik · K. Bakir · A. Oz · M. Kanlikama

Department of Otolaryngology, Head and Neck Surgery,

Gaziantep University, Gaziantep, Turkey

e-mail: tekinbaglam@yahoo.com passage. The clinical, histopathological and therapeutic features of nasal pleomorphic adenomas were discussed.

\section{Case Report}

An 80-year-old woman presented with nasal obstruction, mass visible from both nostrils and nasal deformity. She described one year history of progressive nasal obstruction, occasional nose bleeding and recently worsened nasal deformity. There was no history of visual defect, previous trauma to the nose, weight loss, fever or pain. The patient applied to hospital very late with advanced disease since she was housebound.

Anterior rhinoscopic examination revealed gross nasal mass filling both nasal cavity. Endoscopic examination was not optimum since the mass was obstructing both nasal passages. Cranial nerve examination was normal. There were no palpable lymph nodes of the neck.

Computed tomography (CT) scan showed $7.5 \times 3.5 \mathrm{~cm}$ mass filling nasal cavity, frontal and ethmoid sinuses and expanding medial wall of orbita. It caused erosion of septum, partially anterior part of maxillary sinus. Bilateral osteomeatal complexes were obstructed (Fig. 1).

A punch biopsy of the lesion was performed and histopathologic examination revealed tubular structures within myxoid stroma; epithelial tubular structures infiltrating stroma in some sections. Immunohistochemical staining with Ki67 was performed as proliferation marker. Preoperative diagnosis was compatible with mainly pleomorphic adenoma.

Patient consented for wide surgical excision of the mass by lateral rhinotomy approach due to large tumor size. During surgery, it was seen that the mass was originating from nasal septum and excised totally (Fig. 2). Final 


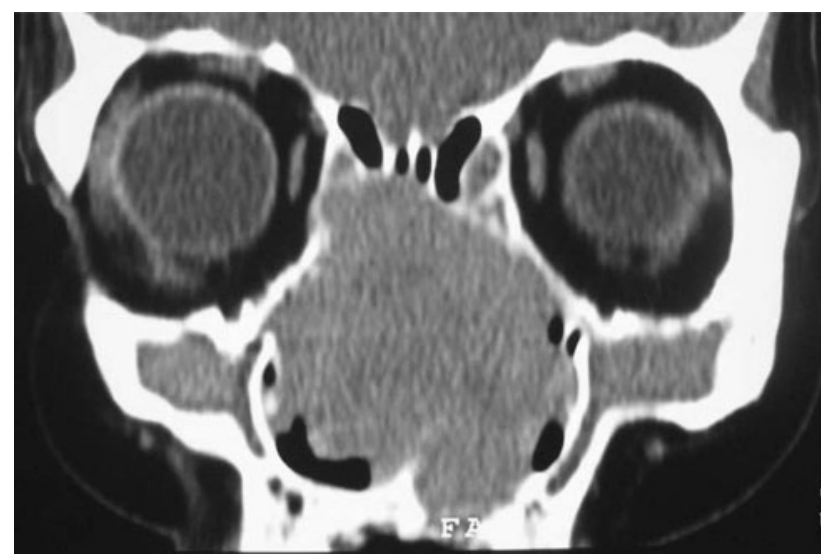

Fig. 1 Coronal computed tomography (CT) showed large intranasal mass

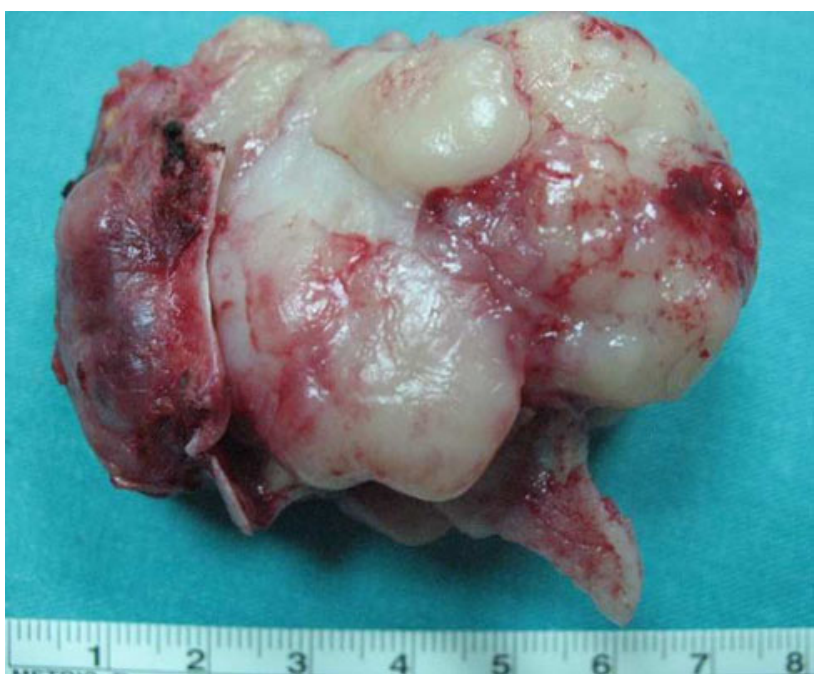

Fig. 2 Gross appearance of surgically excised tumor

histopathological examination confirmed the diagnosis of pleomorphic adenoma (Fig. 3). Patients postoperative course was uneventful and no complication was encountered except mild insicion scarring. No recurrence was present during patient's last visit 18 months after operation.

\section{Discussion}

The vast majority of pleomorphic adenomas arise in the major salivary glands. Those occurring in the minor salivary glands usually arise in the hard or soft palate, although other sites including the nasal cavity, external auditory canal, larynx, pharynx, trachea, and lacrimal glands, have rarely been reported [4]. Nasal pleomorphic adenoma

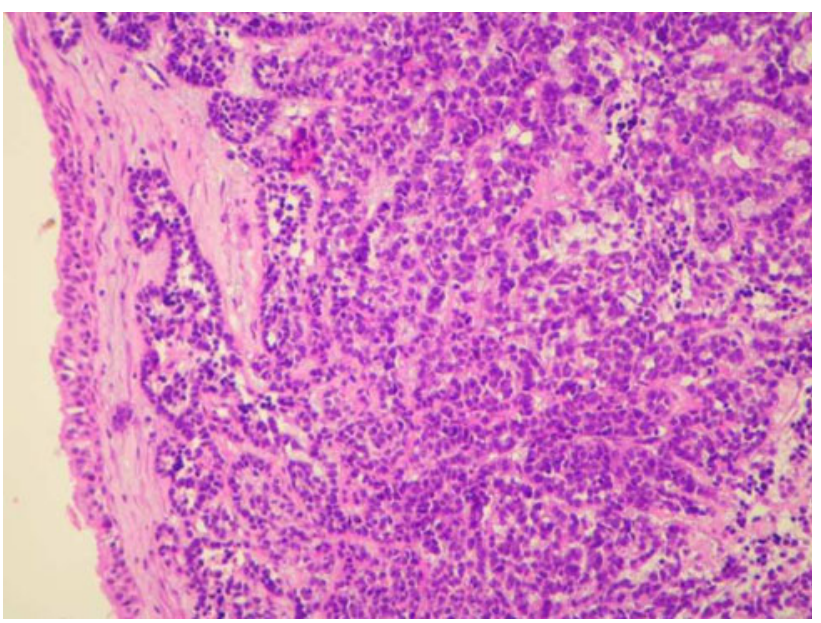

Fig. 3 Histologic section consistent with benign pleomorphic adenoma (hematoxylin-eosin, $\times 100$ ). This section shows greater cellularity and predominant epithelial component and low stromal component; a feature of nasal pleomorphic adenomas

mostly originates from the nasal septum although the vast majority of the minor salivary glands are located in the lateral nasal wall and turbinates [2].

The presenting symptom is usually an unilateral nasal obstruction. Other symptoms may include epistaxis, external deformity, nasal swelling, mucopurulent rhinorrhoea, and epiphora [1,3]. Our patient had an advanced disease due to her delayed application. Both sides of nasal cavity were almost totally occluded by the mass which was visible from both nostrils. Also a nasal deformity was present due to expansive growth of the mass.

Diagnosis of the pleomorphic adenomas mainly rely on pathologic examination. Pleomorphic adenoma constitute both epithelial and mesenchymal component. Nasal pleomorphic adenomas differ from major salivary gland mixed tumours in that they contain greater cellularity and predominant epithelial component with low stromal component and devoid of capsule [3, 5]. Therefore, microscopically they resemble malignant tumors, such as malign mixed tumor. Thus, the diagnosis of the intranasal pleomorphic adenomas may be more challenging. Compango et al. [5] reported 2.5-10\% malignant transformation rate and had a female predominance.

The treatment of intranasal pleomorphic adenoma is surgical excision with wide surgical margin in order to prevent recurrences. Surgical approaches include endonasal endoscopic resection, midfacial degloving, and lateral rhinotomy [1, 2]. Proper surgical technique should be selected according to the size and location of the mass. We prefer external lateral rhinotomy approach considering the tumor size and extension. Endoscopic endonasal resection should be reserved for smaller tumors. 
Recurrence rate of intranasal pleomorphic adenoma is relatively low when compared with pleomorphic adenoma of the major salivary glands and reported as $2.4-10 \%$ $[5,6]$. Clinical follow up is still necessary due to the potential risk of local recurrence.

\section{References}

1. Ünlü HH, Çelik O, Demir MA et al (2003) Pleomorphic adenoma originated from the inferior nasal turbinate. Auris Nasus Larynx $30: 417-420$
2. Tahlan A, Nanda A, Nagarkar $\mathrm{N}$ et al (2004) Pleomorphic adenoma of the nasal septum: a case report. Am J Otolaryngol 25:118-120

3. Gana P, Masterson L (2008) Pleomorphic adenoma of the nasal septum: a case report. J Med Case Rep 2:349

4. Mackle T, Zahirovic A, Walsh M (2004) Pleomorphic adenoma of the nasal septum. Ann Otol Rhinol Laryngol 113(3):210-211

5. Compagno J, Wong RT (1977) Intranasal mixed tumors (pleomorphic adenomas): a clinicopathologic study of 40 cases. Am J Clin Pathol 68(2):213-218

6. Suzuki K, Moribe K, Baba S (1990) A rare case of pleomorphic adenoma of the lateral wall of the nasal cavity, with special reference of statistical observation of pleomorphic adenoma of the nasal cavity in Japan. Nippon Jibiinkoka Gakkai Kaiho 93: 740-745 